\title{
Møller polarimetry with atomic hydrogen targets
}

\author{
E. Chudakov \\ Thomas Jefferson National Accelerator Facility - Newport News, VA 23606, USA
}

ricevuto il 18 Ottobre 2011; approvato il 5 Maggio 2012

pubblicato online il 25 Giugno 2012

\begin{abstract}
Summary. - A proposal to use polarized atomic hydrogen gas as the target for electron beam polarimetry based on the Møller scattering is described. Such a gas, stored in an ultra-cold magnetic trap, would provide a target of practically $100 \%$ polarized electrons. It is conceivable to reach $a \sim 0.3 \%$ systematic accuracy of the beam polarimetry with such a target. Feasibility studies for the CEBAF electron beam have been performed.
\end{abstract}

PACS 29.27.Hj - Polarized beams.

PACS 29.27.Pj - Polarized and other targets.

\section{1. - Motivation}

A better than $0.5 \%$-precise electron beam polarimetry is of critical importance for the next generation of parity violation experiments at Mainz and JLab, discussed by F. Maas and P. Reimer at this Workshop. Compton polarimetry, while potentially accurate enough at $12 \mathrm{GeV}$ has difficulties at low energies and is hardly applicable in the $200 \mathrm{MeV}$ energy range for the proposed experiments at Mainz.

Møller polarimetry does not depend significantly on the beam energy, but the accuracy is limited by the choice of the polarized electron target. Ferromagnetic foils, used so far, provide electron polarization of about $8 \%$. The associated systematic errors were discussed by O. Glamazdin at this Workshop. The dominant errors come from the foil magnetization at saturation (about $0.35 \%$ ) and the Levchuk effect [1] (about $0.3 \%$ ). There are other systematic errors, associated with ferromagnetic targets. The target heating limits the beam current to $3-5 \mu \mathrm{A}$, a factor of 10-20 below the typical currents needed for the experiments. Also, the dead time gives a systematic error.

It seems very attractive to use atomic hydrogen gas, held in an ultra-cold magnetic trap [2], as the source of $100 \%$ polarized electrons. Møller polarimetry with such a target would be free of the accuracy limitations discussed above. The target polarization would be close enough to $100 \%$ and there will be no need to measure it. There will be no Levchuk effect or noticeable dead time. After the idea was originally suggested by the 


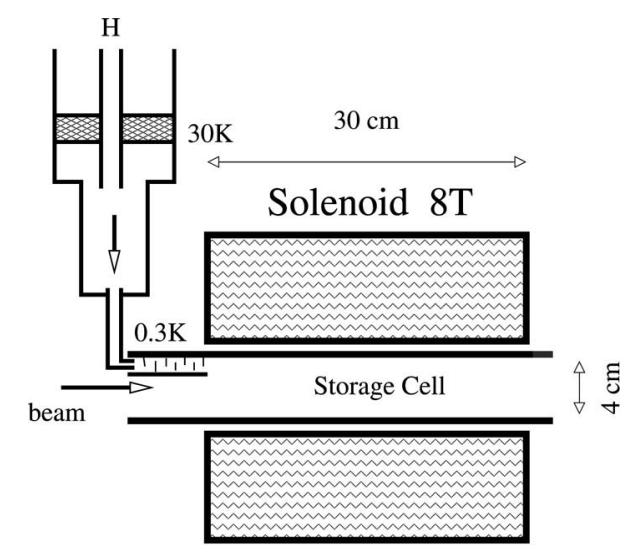

Fig. 1. - A sketch of the storage cell.

same authors [3], the topic is revisited and the calculations verified. Here, the results of the feasibility studies are summarized.

\section{2. - Polarized atomic hydrogen target}

$2 \cdot 1$. Hydrogen atom in magnetic field. - The magnetic field $B_{S}$ and the hyperfine interaction split the ground state of hydrogen into four states with different energies. The low energy states are $|a\rangle=|\downarrow \uparrow\rangle \cdot \cos \theta-|\uparrow \downarrow\rangle \cdot \sin \theta$ and $|b\rangle=|\downarrow \downarrow\rangle$, where the first and second (crossed) arrows in the brackets indicate the electron and proton spin projections on the magnetic field direction. The mixing angle $\theta$ depends on the magnetic field $B_{S}$ and temperature $T$. At $B_{S}=8 \mathrm{~T}$ and $T=0.3 \mathrm{~K}$ the mixing factor is small: $\sin \theta \approx 0.003$. State $|b\rangle$ is $100 \%$ polarized. State $|a\rangle$ is polarized in the same direction as $|b\rangle$ and its polarization differs from unity by $\sim 10^{-5}$.

2². Storage cell. - In a magnetic field gradient, a force $-\nabla\left(\overrightarrow{\boldsymbol{\mu}_{H}} \overrightarrow{\mathbf{B}}\right)$, where $\mu_{H}$ is the atom's magnetic moment, separates the lower and the higher energy states. The lower energy states are pulled into the stronger field, while the higher energy states are repelled from the stronger field. The $0.3 \mathrm{~K}$ cylindrical storage cell, made usually of pure copper, is located in the bore of a superconducting $\sim 8 \mathrm{~T}$ solenoid. The polarized hydrogen, consisting of the low energy states, is confined along the cell axis by the magnetic field gradient, and laterally by the wall of the cell (fig. 1).

At the point of statistical equilibrium, the state population, $p$, follows the Boltzmann distribution $p \propto \exp \left(\mu_{e} B / k T\right)$. Here $\mu_{e}$ is the electron's magnetic moment $\left(\mu_{H} \approx \mu_{e}\right)$ and $k=k_{B}$ is the Boltzmann constant. The cell is mainly populated with states $|a\rangle$ and $|b\rangle$, with an admixture of states $|c\rangle$ and $|d\rangle$ of $\sim 3 \cdot 10^{-16}$. The gas is practically $100 \%$ polarized; a small $\left(\sim 10^{-5}\right)$ oppositely polarized contribution comes from the $|\uparrow \downarrow\rangle$ component of state $|a\rangle$.

The atomic hydrogen density is limited mainly by the process of recombination into $\mathrm{H}_{2}$ molecules (releasing $\sim 4.5 \mathrm{eV}$ ). In case of polarized atoms, the cross section for recombination is strongly suppressed. At moderate gas densities only the surface recombination matters.

A way to reduce the surface recombination on the walls of the storage cell is coating them with a thin film $(\sim 50 \mathrm{~nm})$ of superfluid ${ }^{4} \mathrm{He}$. The cell is filled with atomic hydrogen 
from an RF dissociator. Hydrogen at $30 \mathrm{~K}$ goes from a nozzle into a system of heliumcoated baffles, where it is cooled down to $\sim 0.3 \mathrm{~K}$. Most of the molecules are frozen out in the baffles and do not enter the cell. The gas arrives at the region of a strong field gradient, which separates very efficiently the lower and higher atomic energy states, therefore a constant feeding of the cell does not affect the average electron polarization.

This technique was first successfully applied in 1980 [4], and later a density as high as $3 \cdot 10^{17}$ atoms $/ \mathrm{cm}^{3}$ was achieved [2] in a small volume. So far, the storage cell itself has not been put in a high-intensity particle beam.

For the project being discussed a normal storage cell design can be used, with the beam passing along the solenoid axis (fig. 1). The cell is connected to the beam pipe with no separating windows. The tentative cell parameters are (similar to a working cell [5]): solenoid maximum field of $B_{S}=8 \mathrm{~T}$, solenoid length of $L_{S}=30 \mathrm{~cm}$, cell internal radius of $r_{\circ}=2 \mathrm{~cm}$, cell length of $L_{C}=35 \mathrm{~cm}$ and temperature of $T=0.3 \mathrm{~K}$. The effective length of such a target is about $20 \mathrm{~cm}$. For the guideline, we will consider a gas density of $3 \cdot 10^{15} \mathrm{~cm}^{-3}$, obtained experimentally [6], for a similar design.

$2 \cdot 3$. Gas properties. - By using a calculated value of the hydrogen atoms cross section of $\sigma=42.3 \cdot 10^{-16} \mathrm{~cm}^{2}$ [7] we obtain the mean free path of $\ell=0.57 \mathrm{~mm}$ at a density of $3 \cdot 10^{15} \mathrm{~cm}^{-3}$. The average time, $\tau_{d}$ for a "low field seeking" atom to travel to the edge of the cell, assuming its starting point is distributed according to the gas density, is $\left({ }^{1}\right)$ : $\tau_{d} \approx 0.7 \mathrm{~s}$. This is the cleaning time for an atom with opposite electron spin, should it emerge in the cell and if it does not recombine before. The escape time depends on the initial position of the atom, going from $\sim 1 \mathrm{~s}$ at $z=0$ to $0.1 \mathrm{~s}$ at $z=8 \mathrm{~cm}$. The average wall collision time is about $0.5 \mathrm{~ms}$.

$\mathbf{2}$ 4. Gas lifetime in the cell. - The processes which determine the average lifetime of a "high field seeking" atom in the cell are well understood and the values measured have been reproduced by calculations. At a density of $3 \cdot 10^{15} \mathrm{~cm}^{-3}[6]$ the lifetime is $\sim 1 \mathrm{~h}$.

2.5. Unpolarized contamination in the absence of beam. - The contamination by hydrogen molecules and excited atoms was estimated to be small $\left(\sim 10^{-5}\right)$. The upper limit on this contamination can be derived from the gas lifetime. A larger contamination may come from the residual gas in the beam pipe $\left(\sim 10^{-3}\right)$. This contribution can be measured by comparing the counting rate of the polarimeter at different hydrogen densities. Another possibility is to measure the location of the scattering point along the target. The associated uncertainty will be reduced to $<0.5 \cdot 10^{-3}$.

\section{3. - Beam impact on storage cell}

We have considered various impacts the $\mathcal{I}_{b}=100 \mu \mathrm{A}$ CEBAF beam can inflict on the storage cell. The beam consists of short bunches with $\tau=\sigma_{T} \approx 0.5 \mathrm{ps}$ at a $\mathcal{F}=$ $499 \mathrm{MHz}$ repetition rate. The beam spot has a size of about $\sigma_{X} \approx \sigma_{Y} \sim 0.1 \mathrm{~mm}$. The most important depolarization effects we found are: a) gas depolarization by the RF electromagnetic radiation of the beam $\left(\sim 3 \cdot 10^{-5}\right)$; b) contamination from free electrons and ions $\left(\sim 10^{-5}\right)$.

$\left({ }^{1}\right)$ This time was estimated using simulation, taking into account the gas density distribution along $z$ and the repelling force in the magnetic field gradient. 


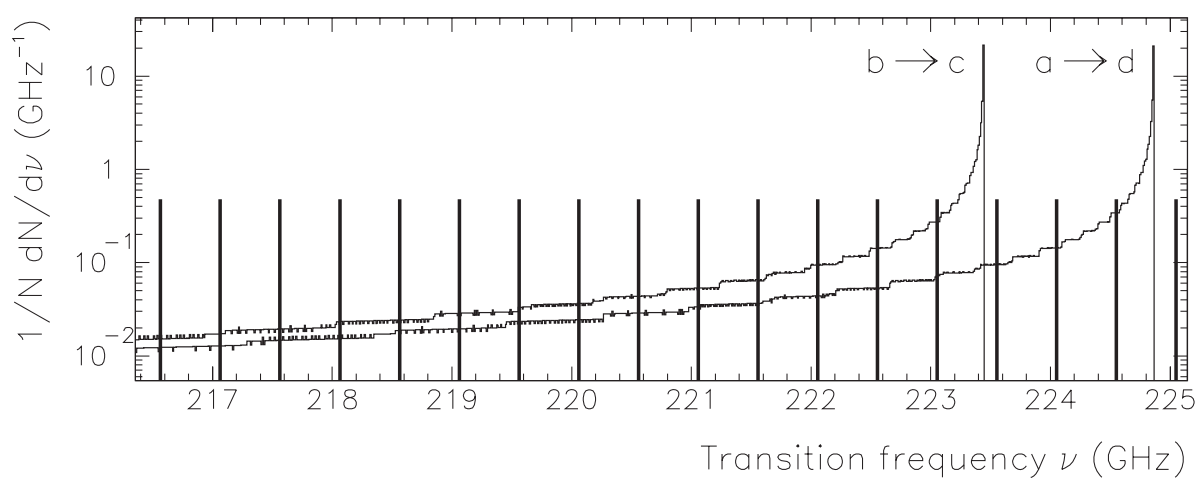

Fig. 2. - Simulated spectra of the transitions on the axis of the hydrogen trap with the maximum field of $8.0 \mathrm{~T}$. The density of atoms depends on the field as $\exp \left(-\mu_{e} B / k T\right)$. The two curves show $\frac{1}{N} \mathrm{~d} N / \mathrm{d} \nu_{a d}$ and $\frac{1}{N} \mathrm{~d} N / \mathrm{d} \nu_{b c}$ - the relative number of atoms which can undergo $|a\rangle \rightarrow|d\rangle$ and $|b\rangle \rightarrow|c\rangle$ transitions at the given frequency, per one GHz. The resonant structure of the spectral function of the beam-induced electromagnetic field is shown as a set of vertical bars, $499 \mathrm{MHz}$ apart.

3`1. Beam RF generated depolarization. - The electromagnetic field of the beam has a circular magnetic field component, which couples to the $|a\rangle \rightarrow|d\rangle$ and $|b\rangle \rightarrow|c\rangle$ transitions. The transition frequency depends on the value of the local magnetic field in the solenoid and for the bulk of the gas ranges from 215 to $225 \mathrm{GHz}$. The spectral density function of the magnetic field can be presented in the form of Fourier series with the characteristic frequency of $\omega_{\circ}=2 \pi \mathcal{F}$. The resonance lines of the spectrum (a reflection of the $499 \mathrm{MHz}$ repetition rate) populate densely the transition range (see fig. 2). The induced transition rate depends on the gas density at a given transition frequency. This rate was calculated taking into account the beam parameters and the field map of a realistic solenoid. Provided that the field of the solenoid is fine tuned to avoid the transition resonances for the bulk of the gas in the cell (see fig. 2), the depolarization described has the following features: the transition rate is proportional to $\mathcal{I}_{b}^{2}$; the average rate of each of the two transitions is about $0.5 \cdot 10^{-4}$ of the target density per second; at the center around the beam the full transition rate is about $6 \%$ of the density per second.

By taking into account that each resonance line presented in fig. 2 corresponds to a certain value of the solenoid field and, therefore, affects the gas at a certain $z$, we obtained the result that the average depolarization in the beam area will be reduced to about $\sim 0.3 \cdot 10^{-4}$ by the lateral gas diffusion and by the escape of the "low field seeking" atoms from the storage cell.

3.2. Contamination by free electrons and ions. - The beam would ionize per second about $20 \%$ of the atoms in the cylinder around the beam spot. The charged particles would not escape the beam area due to diffusion, as the neutral atoms would do, but will follow the magnetic field lines, parallel to the beam. An elegant way to remove them is to apply a relatively weak $\sim 1 \mathrm{~V} / \mathrm{cm}$ electric field perpendicular to the beam. The charged particles will drift at a speed of $\overrightarrow{\mathbf{v}}=\overrightarrow{\mathbf{E}} \times \overrightarrow{\mathbf{B}} / B^{2} \sim 12 \mathrm{~m} / \mathrm{s}$ perpendicular to the beam and leave the beam area in about $20 \mu \mathrm{s}$. This will reduce the average contamination to a $10^{-5}$ level. Designing appropriate electrodes for the He-coated cell will require R\&D efforts. 


\section{4. - Application of the atomic target to Møller polarimetry}

With a $50 \mu \mathrm{A}, 90 \%$ polarized beam, we expect to reach a $1 \%$ statistical accuracy (relative) in about $15 \mathrm{~min}$. The polarization measurements can go continuously with the main data taking, providing that the beam optics is flexible enough to satisfy the requirements of the experiment and the polarimeters.

There is no obvious way to measure directly the polarization of the hydrogen atoms in the beam area. The contamination from the residual gas is measurable. The rest (about $0.01 \%$ ) relies on calculations. The impact of the most important of these contributions can be studied by deliberately increasing the effect. The beam RF-induced transitions can be increased by a factor of $\sim 70$, by fine tuning of the solenoid magnetic field. The contribution from the charged particles in the beam area can be varied by a factor up to $\sim 10^{4}$, by changing the cleaning electric field.

The known systematic errors, associated with the present high-field Hall A polarimeter, when added in quadrature give a total systematic error of about $0.7 \%$. Scaling these errors to the hydrogen target option reduces the total error to about $0.2 \%$. It is conceivable that other systematic errors now unnoticed will show up, of the similar scale, increasing the total error to about $0.3 \%$.

\section{5. - Conclusion}

The considerations above show that a stored, longitudinally electron-spin-polarized atomic hydrogen can be used as a pure, $100 \%$ electron polarized gas target. The polarized hydrogen gas should be stable in the presence of a $100 \mu \mathrm{A}$ CEBAF beam. A Møller polarimeter, equipped with such a target could provide a superb systematic accuracy of about $0.3 \%$, while providing a $1 \%$ statistical accuracy in about $15 \mathrm{~min}$ of running at a beam current of $50 \mu \mathrm{A}$.

Notice: Authored by Jefferson Science Associates, LLC under U.S. DOE Contract No. DE-AC05-06OR23177. The U.S. Government retains a non-exclusive, paid-up, irrevocable, world-wide license to publish or reproduce this manuscript for U.S. Government purposes.

\section{REFERENCES}

[1] Levchuk L., Nucl. Instrum. Methods A, 345 (1994) 496.

[2] Silvera I. and Walraven J., Progress in low temperature physics, Ser. Phys., Vol. 10 (North-Holland) 1986, pp. 139-370.

[3] Chudakov E. and Luppov V., IEEE Trans. Nucl. Sci., 51 (2004) 1533.

[4] Silvera I. F. and Walraven J. T. M., Phys. Rev. Lett., 44 (1980) 164.

[5] Roser T., Crabb D., Kaufman W., Raymond R., Stewart J. et al., Nucl. Instrum. Methods A, 301 (1991) 42.

[6] Mertig M., Luppov V. G., Roser T. and Vuaridel B., Rev. Sci. Instrum., 62 (1991) 251.

[7] Miller M. D. and Nosanow L. H., Phys. Rev. B, 15 (1977) 4376. 